\title{
Dipolar Order in Molecular Fluids: II. Molecular Influence
}

\author{
Per Linse • Gunnar Karlström
}

Received: 25 March 2011 / Accepted: 8 September 2011 / Published online: 7 October 2011

(C) The Author(s) 2011. This article is published with open access at Springerlink.com

\begin{abstract}
The influence on the short-range packing in dipolar fluids by molecular shape and by additional higher order electrostatic moments has been investigated by molecular dynamic simulations. The dipole polarization was found to decrease as the particles were elongated parallel to the dipole and to increase for elongation perpendicular to the dipole, eventually forming a nematic order. The addition of a quadrupole lead to a reduction of the polarization, and the influence of an axial octupole was weaker and more complex. Both a decrease and an increase of the polarization is possible depending on the relative dipoledipole and octupole-octupole interaction strengths and the relative direction of the symmetry axes of the moments. These observations were attributed to the different parity of a dipole and a quadrupole and the same parity of a dipole and an axial octupole under reflection. In addition, further insights into the formation of dipole polarization were obtained. Short polar and long equatorial radii and strong dipole-dipole interaction are particle properties that promote a fluid with a high dipole polarization.
\end{abstract}

Keywords Dipolar order · Molecular fluid · Molecular shape $\cdot$ Electrostatic multipole moment

\section{Introduction}

Investigations of the dielectric behavior of molecular media have been the subject for over 150 years (see references given in Ref. [1]); yet a deep understanding of the many-body phenomena giving rise to the collective dipolar polarization is still incomplete. Such an understanding of the relation between the molecular properties and interactions, on one hand, and the spontaneous and fluctuating polarization, on the other hand, is of central importance (i) to rationalize the solvation of ions in media and manipulate properties of solvent

P. Linse $(\bowtie)$

Division of Physical Chemistry, Department of Chemistry, P.O. Box 124, 22100 Lund, Sweden

e-mail: per.linse@fkem1.lu.se

G. Karlström

Division of Theoretical Chemistry, Department of Chemistry, P.O. Box 124, 22100 Lund, Sweden 
molecules to achieve desired dielectric properties and (ii) to relate orientational correlations of particles with their structure and dipole moment with the goal to fabricate particles with more complex non-central forces.

The two most used systems for investigating properties of simple dipolar fluid involves dipolar hard-sphere and dipolar Lennard-Jones (Stockmayer) particles. The former system has been investigated by Verlet and Weis [2], Patey and Valleau [3], Levesque et al. [4], Ladd [5] and the latter by Wang [6], McDonald [7], and Verlet and Weis [2]. Particles having both a dipole and a quadrupole have been considered by Patey and Valleau [3].

In the preceding paper [1] of this issue, also referred to as paper I, we have by simulations investigated how the dipole polarization of a fluid composed of spherical particles with ideal dipole moments is affected by manipulations of the radial and of the angular spatial correlations among the particles. In the present contribution, we will examine how the polarization of a dipolar fluid is affected by its molecular properties through (i) shape deviation from spherical symmetry and (ii) deviation from a pure electrostatic dipole-dipole interaction. The former is achieved by replacing the spherical excluded volume by two and slightly displaced spheres, and this displacement will be made both parallel and perpendicular to the dipole. The electrostatic interaction is modulated by adding either of the next two moments of an electrostatic multipole expansion, i.e., a quadrupole or an octupole.

Previously, Morriss [8] examined the dielectric constant in fluids of polar hard dumbbells particles and, in particular, found that the dielectric permittivity was reduced as the dumb-bells were extended parallel to the dipole vector. Furthermore, Patey and Valleau [3] found that the dielectric permittivity of a dipolar hard-sphere solution was reduced as a quadrupole moment was added to the particles. The present work confirms these trends and offers a more detailed investigation of the molecular origin of the variation of the dielectric polarization and concomitant permittivity.

\section{Model and Method}

Consider a model fluid composed of $N$ particles in a cubic volume $V$ at temperature $T$. The potential energy $U$ of the fluid is assumed to be pairwise additive according to

$$
U=\sum_{i=1}^{N-1} \sum_{j=i+1}^{N} u_{i j}\left(r_{i j}, \Omega_{i}, \Omega_{j}\right)
$$

where $\mathbf{r}_{i j}=\mathbf{r}_{i}-\mathbf{r}_{j}, r_{i j}=\left|\mathbf{r}_{i j}\right|$, and $\Omega_{i}$ collectively represents the two polar coordinates $\theta_{i}$ and $\varphi_{i}$ describing the orientation of particle $i$ with respect to $\mathbf{r}_{i j}$. The interaction between particles $i$ and $j$ is composed of a short-range $u_{i j}^{\mathrm{SR}}\left(r_{i j}, \Omega_{i}, \Omega_{j}\right)$ and an electrostatic $u_{i j}^{\text {elec }}\left(r_{i j}, \Omega_{i}, \Omega_{j}\right)$ interaction according to

$$
u_{i j}\left(r_{i j}, \Omega_{i}, \Omega_{j}\right)=u_{i j}^{\mathrm{SR}}\left(r_{i j}, \Omega_{i}, \Omega_{j}\right)+u_{i j}^{\mathrm{elec}}\left(r_{i j}, \Omega_{i}, \Omega_{j}\right) .
$$

We will consider three types of particles. They are (A) particles with centerosymmetric short-range potential (referred to as reference particles), (B) particles with noncenterosymmetric short-range potential (shape-asymmetric particles), and (C) particles with a multipole of higher order than a dipole (particles with modified electrostatic interaction).

(A) As to reference particles (labeled Ref), the short-range interaction is taken to be a single Lennard-Jones (LJ) potential according to

$$
u_{i j}^{\mathrm{SR}}\left(r_{i j}, \Omega_{i}, \Omega_{j}\right)=u_{i j}^{\mathrm{LJ}}\left(r_{i j}\right)
$$




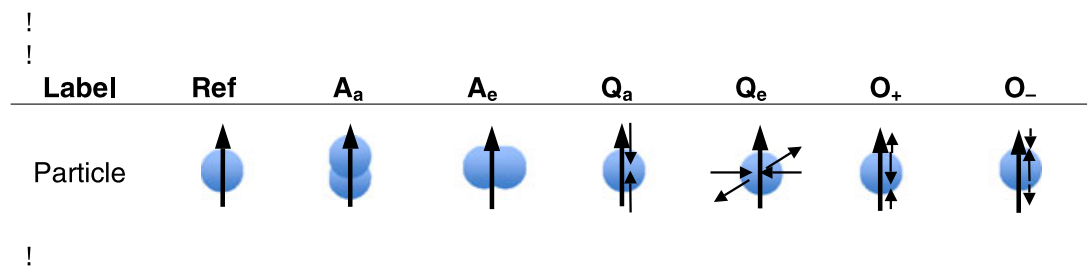

Fig. 1 (Color online) Illustration of the reference particle (Ref), shape-asymmetric particles $\left(A_{a}\right.$ and $\left.A_{e}\right)$, and particles with modified electrostatic interactions $\left(\mathrm{Q}_{\mathrm{a}}, \mathrm{Q}_{\mathrm{e}}, \mathrm{O}_{+}\right.$, and $\left.\mathrm{O}_{-}\right)$investigated showing the $\mathrm{LJ}$ centers (blues spheres), dipoles (thick arrow), and higher order multipoles obtained by a combination of several dipoles (thin solid arrows). See Table 1 for further details

where

$$
u_{i j}^{\mathrm{LJ}}\left(r_{i j}\right)=4 \varepsilon\left[\left(\frac{\sigma}{r_{i j}}\right)^{12}-\left(\frac{\sigma}{r_{i j}}\right)^{6}\right]
$$

with $\sigma$ being the size and $\varepsilon$ the interaction parameter of the LJ potential. Furthermore, the electrostatic interaction was taken to be a dipole-dipole potential according to

$$
u_{i j}^{\text {elec }}\left(r_{i j}, \Omega_{i}, \Omega_{j}\right)=u_{i j}^{\mathrm{dip}}\left(r_{i j}, \Omega_{i}, \Omega_{j}\right)
$$

where

$$
u_{i j}^{\mathrm{dip}}\left(r_{i j}, \Omega_{i}, \Omega_{j}\right)=\frac{1}{4 \pi \varepsilon_{0}}\left[\frac{\boldsymbol{\mu}_{i} \cdot \boldsymbol{\mu}_{j}}{r_{i j}^{3}}-\frac{3\left(\boldsymbol{\mu}_{i} \cdot \mathbf{r}_{i j}\right)\left(\boldsymbol{\mu}_{j} \cdot \mathbf{r}_{i j}\right)}{r_{i j}^{5}}\right]
$$

with $\boldsymbol{\mu}_{i}$ denoting the dipole (characterized by magnitude and direction) of particle $i$, see Fig. 1.

(B) Regarding shape-asymmetric particles, each particle were composed of two LJ sites with the separation $d$, and the LJ interaction parameter $\varepsilon$ were reduced by the factor 4 , making the $\mathrm{LJ}$ interaction for the fluid with shape-asymmetric particles to merge that of the reference fluid as $d \rightarrow 0$. Thus, the short-range interaction becomes

$$
u_{i j}^{\mathrm{SR}}\left(r_{i j}, \Omega_{i}, \Omega_{j}\right)=\sum_{\substack{k=1 \\ k \in i}}^{2} \sum_{\substack{l=1 \\ l \in j}}^{2} u_{k l}^{\mathrm{LJ}}\left(r_{k l}\right)
$$

with $r_{k l}$ denoting the distance between site $k$ belonging to particle $i$ and site $l$ belonging to particle $j$. The electrostatic interaction is still given by the dipole-dipole potential, see (5) and (6). Two types of shape-asymmetric particles were considered: particle elongation parallel to the dipole direction $\left(\mathrm{A}_{\mathrm{a}}\right)$ and perpendicular to the dipole direction $\left(\mathrm{A}_{\mathrm{e}}\right)$, see Fig. 1.

(C) In the case of modified electrostatic interaction, the particles contained, in addition to the dipole, (i) a quadrupole or (ii) an octupole, and the short-range interaction remained spherical symmetric, see (3) and (4). Two different types of quadrupoles were used, viz. an axial $\left(\mathrm{Q}_{\mathrm{a}}\right)$ and an equatorial $\left(\mathrm{Q}_{\mathrm{e}}\right)$ quadrupole. Finally, a single axial octupole was employed with two different directions were considered: parallel $\left(\mathrm{O}_{+}\right)$and antiparallel $\left(\mathrm{O}_{-}\right)$ to the dipole, see Fig. 1. Generally, multipole-multipole interactions become more angular dependent and decays faster with increasing separation as the number of poles of the multipoles increases [9]. Expressions for interaction energies and their ratios between relevant multipoles are given in the Appendix. 
Table 1 Particles investigated ${ }^{\mathrm{a}}$

\begin{tabular}{llllll}
\hline Label & Description & $d(\AA)^{\mathrm{b}}$ & $\mu_{\mathrm{q}}(e \AA)^{\mathrm{c}}$ & $\left(Q_{\mathrm{xx}}, Q_{\mathrm{yy}}, Q_{\mathrm{zz}}\right)^{\mathrm{d}} O_{\mathrm{zzz}}$ \\
\hline Ref & 1 LJ site & - & - & - & - \\
$\mathrm{A}_{\mathrm{a}}$ & 2 LJ sites (parallel) & $0.1,0.2,0.4,1.0$ & - & - & - \\
$\mathrm{A}_{\mathrm{e}}$ & 2 LJ sites (perpendicular) & $0.1,0.2,0.4,1.0-$ & - & - \\
$\mathrm{Q}_{\mathrm{a}}$ & Addition of axial Q & - & $0.2,0.5,1.0,2.0 \quad(-1,-1,2) \mu_{\mathrm{q}} \delta$ & - \\
$\mathrm{Q}_{\mathrm{e}}$ & Addition of equatorial Q & - & $0.2,0.5,1.0,2.0 \quad(3,-3,0) \mu_{\mathrm{q}} \delta$ & - \\
$\mathrm{O}_{+}$ & Addition of axial O (parallel) & - & $1.0,2.0,4.0,8.0-$ & $2\left(\mu_{\mathrm{q}} \delta\right)^{2}$ \\
$\mathrm{O}_{-}$ & Addition of axial O (antiparallel) & - & $1.0,2.0,4.0,8.0-$ & $-2\left(\mu_{\mathrm{q}} \delta\right)^{2}$ \\
\hline
\end{tabular}

${ }^{\mathrm{a}}$ Quadrupoles and octupoles were built up by a combination of several dipoles $\boldsymbol{\mu}_{\mathrm{q}}$ as illustrated in Fig. 1. Those ones off-center were placed $\delta=0.1 \AA$ from the center. The contributions from the concomitant higher order moments to the interaction energy were negligible

${ }^{\mathrm{b}}$ Distance between two LJ sites

${ }^{\mathrm{c}}$ Dipole moment of dipoles forming the quadrupoles and octupoles

${ }^{\mathrm{d}}$ Irreducible Cartesian quadrupole moments

Throughout, we have used the Lennard-Jones parameters $\sigma=2.8863 \AA$ and $\varepsilon=$ $1.97023 \mathrm{~kJ} / \mathrm{mol}$ as well as the dipole moment $\mu=0.34397 e \AA$ ( $=1.651 \mathrm{D}$, cf. $1.9-2.9 \mathrm{D}$ for water in different states) at the temperature $T=315.8 \mathrm{~K}$ as used in previous investigations [10]. In reduced units, the reference fluid is characterized by $\mu^{*} \equiv \mu /\left(4 \pi \varepsilon_{0} \varepsilon \sigma^{3}\right)^{1 / 2}=1.863$ and $T^{*} \equiv k T / \varepsilon=1.333$. In the fluids with shape-asymmetric particles, we have used values of $d$ up to $1.0 \AA$.

The quadrupoles and octupoles were built up of combinations of dipoles $\boldsymbol{\mu}_{\mathrm{q}}$ placed at the center and at the distance $\delta=0.1 \AA$ from the center of the particles. The electrostatic interactions involving quadrupoles and octupoles were obtained by summing over sets of these dipoles. The use of a set of ideal dipoles will, in addition to the quadrupoles or octupoles, also generate multipoles of higher order than quadrupoles or octupoles, respectively. However, since $\delta$ is much smaller than the particle size, $\sigma \approx 3 \AA$, these higher-order contributions are negligible. Values of $d$ and $\boldsymbol{\mu}_{\mathrm{q}}$ as well as the irreducible Cartesian quadrupole tensor $\mathbf{Q}$ and the octupole tensor element $O_{\text {zzz }}$ are compiled in Table 1 .

Simulations have been made in the NTV ensemble at the number density $\rho=N / V=$ 0.038446 ( $\left.\rho^{*} \equiv \rho \sigma^{3}=0.9244\right)$ using $N=1000$ and 3000 particles. The results were statistically identical for the two values of $N$, and only those for $N=3000$ will be given. As the volume and the number of particles were kept constant, the particle volume fraction for fluids with shape-asymmetric particles is slightly increased at increasing $d$. The long-range interaction was handled using the Ewald summation. The MD simulations were performed using the velocity Verlet algorithm with the orientations described by quaternions using a time step $\Delta t=0.001 \mathrm{ps}$, corresponding to a reduced time step $\Delta t^{*} \equiv \Delta t\left(m \sigma^{2} \varepsilon\right)^{1 / 2}=0.0011$, where the mass $m=18 \mathrm{~g} \mathrm{~mol}^{-1}$ was used. The particles were treated as spherical tops with the components of the moment of inertia $I_{\mathrm{xx}}=I_{\mathrm{yy}}=I_{\mathrm{zz}}=1 \mathrm{~g} \mathrm{\AA}^{2} \mathrm{~mol}^{-1}$. After equilibration, each simulation involved $10^{5}$ time steps, hence extending over $t_{\text {sim }}=100$ ps. Berendsen's approach [11] of coupling of the system to an external bath with a time-coupling constant of $100 \Delta t$ to preserve the temperature $T$ was used. This weak coupling only suppresses the potential energy drift and does not affect the dynamics of the system. The integrated Monte Carlo/molecular dynamics/Brownian dynamics simulation package MOLSIM [12] for molecular systems was employed throughout. 
Fig. 2 (Color online) (a) Center-of-mass-center-of-mass radial distribution function $g(r)$ and (b) normalized angular probability functions $P[\cos (\theta)]$ and $P[\cos (\psi)]$ of fluids containing reference particles (green) and $\mathrm{A}_{\mathrm{a}}$ (blue) and $\mathrm{A}_{\mathrm{e}}$ (red) particles with $d=0.4 \AA$. In (b), the insert shows the definitions of the angles $\theta$ and $\psi$
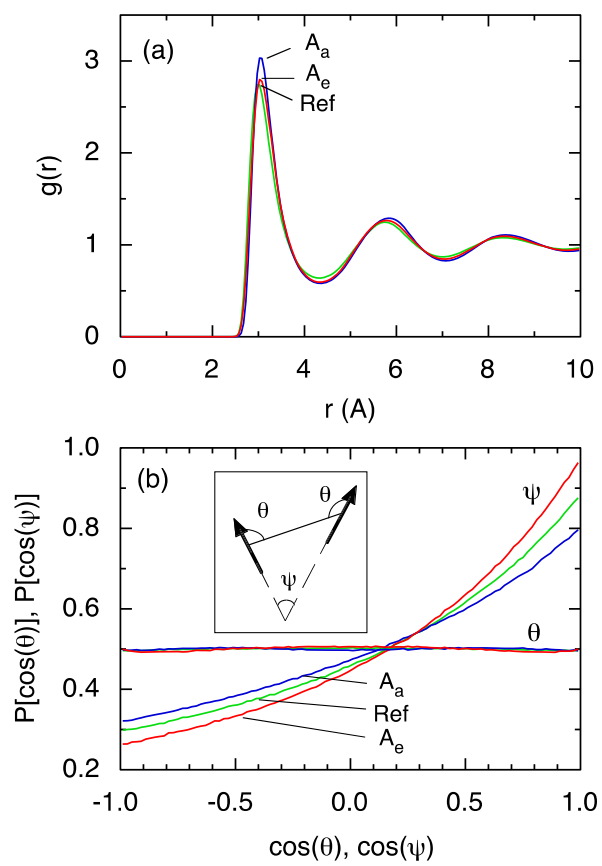

\section{Analyses}

The fluids with particles of the different asymmetries were analyzed using (i) radial distribution function $g(r)$, (ii) normalized angular probability functions $P(\cos \theta)$ and $P(\cos \psi)$, (iii) two-dimensional radial-angular distribution function $g(r, \cos \theta)$, (iv) distance-dependent Kirkwood function $G_{\mathrm{k}}(r)$, (v) dipolar domain analyses, and (vi) two-dimensional radialangular intrinsic polarization function $\mathcal{P}(r, \cos \theta)$.

The radial distribution function $g(r)$ is conventionally defined as

$$
g(r)=\frac{\rho(r)}{\bar{\rho}}
$$

where $\rho(r)$ is the local number density at the distance $r$ from a particle and $\bar{\rho}=N / V$ is the average number density of the fluid.

The normalized angular probability function $P(\cos \theta)$ denotes the probability of finding a neighboring particle in a direction forming the angle $\theta$ with respect to the dipole of the central particle (see insert of Fig. 2(b)). Thus, $\cos \theta=1$ denotes the forward dipole direction, $\cos \theta=-1$ the reverse dipole direction, and $\cos \theta=0$ a direction perpendicular to the dipole. Furthermore, $\psi$ denotes the angle formed by the directions of the two dipoles. In these angular analyses, averages are made over particles laying within the first maximum of the radial distribution function, $r<r^{\prime}=4.2 \AA$.

The radial-angular distribution function $g(r, \cos \theta)$ is defined according to

$$
g(r, \cos \theta)=\frac{\rho(r, \cos \theta)}{\bar{\rho}}
$$

where $\rho(r, \cos \theta)$ is the local number density at the distance $r$ and the angle $\theta$ from a particle. The radial distribution function $g(r)$ is related to the radial-angular distribution function 
$g(r, \cos \theta)$ by the angular integration

$$
g(r)=\frac{1}{2} \int_{-1}^{1} g(r, \cos \theta) d(\cos \theta)
$$

and the angular probability function $P(\cos \theta)$ to the radial-angular distribution function $g(r, \cos \theta)$ by the radial integration

$$
P(\cos \theta)=\left(\frac{3}{r^{\prime 3}}\right) \int_{0}^{r^{\prime}} P(\cos \theta, r) r^{2} d r
$$

with

$$
P(\cos \theta, r)=\frac{g(r, \cos \theta)}{\frac{1}{2} \int_{-1}^{1} g(r, \cos \theta) d(\cos \theta)}
$$

where the intermediate angular probability function $P(\cos \theta, r)$ denotes the probability of the angle $\theta$ given the distance $r$.

One of the intriguing properties of dipolar fluids is the formation of nematic (ferroelectric) domains and phases. Consider the measure of the directional ordering of the particles in a spherical volume $V^{\prime}$ centered on dipole $i$ with radius $r$ at according to

$$
\widehat{G}_{\mathrm{k}, i}(r)=\frac{1}{\mu^{2}}\left(\mu_{i} \cdot \sum_{j \in V^{\prime}} \mu_{j}\right)
$$

(hat denotes that a quantity is given for a configuration). If the dipoles of the particles are orientationally uncorrelated, we have $\widehat{G}_{\mathrm{k}, i}(r)=1$, whereas if they are positively correlated $\widehat{G}_{\mathrm{k}, i}(r)>1$ and if they are negatively correlated $\widehat{G}_{\mathrm{k}, i}(r)<1$. The time average of $\widehat{G}_{\mathrm{k}, i}(r)$ will be denoted $G_{\mathrm{k}, i}(r)$. Moreover, for a homogeneous fluid we can form the average of $G_{\mathrm{k}}(r)$ over all the particles according to

$$
G_{\mathrm{k}}(r)=\frac{1}{N} \sum_{i=1}^{N} G_{\mathrm{k}, i}(r)
$$

which often is referred to as the distance-dependent Kirkwood function. After the limit $V / V^{\prime} \rightarrow \infty$ followed by the limit $r \rightarrow \infty$, the distance-dependent Kirkwood function $G_{\mathrm{k}}(r)$ becomes the Kirkwood $g_{\mathrm{K}}$-factor.

The first non-trivial maximum of $\widehat{G}_{\mathrm{k}, i}(r), \widehat{G}_{\mathrm{k}, i}^{\mathrm{max}}$, was used as a measure of the strength of the instantaneously dipole ordering around particle $i$, and the radius at which $\widehat{G}_{\mathrm{k}, i}(r)$ displayed its first maximum, $R_{\mathrm{max}, i}$, as the measure of the extension of this domain of orientationally ordered dipoles. From $G_{\mathrm{k}, i}^{\max }(i=1, \ldots, N)$, a set of ordered domains containing particles with ordered dipoles was constructed. Normalized probability distribution functions $P\left(G_{\mathrm{k}}^{\max }\right)$ and $P\left(R_{\max }\right)$ as well as correlation plots of $G_{\mathrm{k}}^{\max }$ versus $R_{\max }$ were formed by using the largest domains of this set. For further details, see Ref. [13].

Beside the local density, we will also consider the local polarization. Let $\mathcal{P}(r, \cos \theta)$ denote the local polarization at distance $r$ and the angle $\theta$ from a particle with respect to the dipole vector of the particle. Then we, have the relation between the distance-dependent Kirkwood function and the local polarization according to

$$
G_{\mathrm{k}}(r)=\int_{0}^{r} \int_{0}^{\pi} \mathcal{P}\left(r^{\prime}, \cos \theta\right)\left[d(\cos \theta) r^{\prime 2} d r^{\prime}\right]
$$


with $\mathcal{P}(r, \cos \theta)$ formally given by

$$
\mathcal{P}(r, \cos \theta)\left[r^{2} d r d(\cos \theta)\right]=\frac{1}{\mu^{2}}\left(\mu_{i} \cdot \sum_{j \in d V} \mu_{j}\right)
$$

where $d V=r^{2} d r d(\cos \theta)$ denotes a volume element around the point $(r, \cos \theta)$. By dividing $\mathcal{P}(r, \cos \theta)$ by the local number density $\rho(r, \cos \theta)$, we get the density normalized (intrinsic) local polarization $\mathfrak{p}(r, \cos \theta)$ according to

$$
\mathfrak{p}(r, \cos \theta)=\frac{\mathcal{P}(r, \cos \theta)}{\rho(r, \cos \theta)}
$$

with the limits $-1 \leq \mathfrak{p} \leq 1$.

The sampling of the various distribution functions was made using histograms. The width of the histograms were (i) $0.05 \AA$ for radial distribution functions, (ii) 0.02 for angular distribution functions, (iii) $0.1 \AA$ and 0.1 for radial-angular distribution functions and intrinsic polarization functions, (iv) $0.2 \AA$ for distance-dependent Kirkwood functions, and (v) 5 and $0.2 \AA$ for distribution functions of dipole domains. The radial-angular distribution functions and intrinsic polarization functions are represented by contour plots with iso- $g$ curves at every 0.2 and iso-polarization curves at every 0.1 , respectively.

\section{Results}

\subsection{Shape Asymmetry}

We will start by considering shape-asymmetric particles. As shown in Fig. 1, these particles are elongated by replacing the single LJ site by two LJ sites separated by the distance $d$ with the elongation direction either parallel $\left(\mathrm{A}_{\mathrm{a}}\right)$ or perpendicular $\left(\mathrm{A}_{\mathrm{e}}\right)$ to the dipole. The separation has been varied between $d=0.1$ and $1.0 \AA$ (cf. the LJ diameter $\sigma \approx 3 \AA$ ).

Simple dipolar fluids are conventionally investigated by using the radial distribution function and the two angular probability functions as described in the previous section. Figure 2 shows the center-of-mass-center-of-mass radial distribution function $g(r)$, the angular probability functions $P(\cos \theta)$ involving the angle $\theta$ formed by the interparticle vector $\mathbf{r}_{i j}$ and the dipole vector $\boldsymbol{\mu}_{i}$, and $P(\cos \psi)$ involving the angle $\psi$ formed by the dipole vectors $\boldsymbol{\mu}_{i}$ and $\boldsymbol{\mu}_{j}$. Both probability functions are averaged over nearest neighbor particles $j$ of the central particle $i$. Data are given for the reference fluid and the two different types of shapeasymmetric particles with $d=0.4 \AA$; hence $d / \sigma \approx 1 / 7$.

The radial distribution functions of the three fluids are similar (Fig. 2(a)), which might be expected from the small $d / \sigma$ ratio. However, a closer inspection shows that the fluids with the two shape-asymmetric particles display a slightly larger order. An increased order is expected from the increase of the effective particle volume with increasing $d$, while the number density is the same for the fluids. The radial distribution functions of the two shape-asymmetric particles are very similar except around the first maximum where the peak is higher for the case where the particle elongation is parallel to the dipole. The angular probability functions $P(\cos \theta)$ of the three fluids are equal and basically constant $(=0.5)$ (Fig. 2(b)), whereas $P(\cos \psi)$ increases with $\cos \psi$ demonstrating a preferentially net parallel orientation of the dipoles of adjacent particles. The degree of net preferential parallel dipole orientation is larger for the perpendicular case $\left(\mathrm{A}_{\mathrm{e}}\right)$ and smaller for the parallel 
Fig. 3 (Color online)

Distance-dependent Kirkwood

function $G_{\mathrm{k}}(r)$ of fluids

containing reference particles

(green) and $\mathrm{A}_{\mathrm{a}}$ (solid curves) and

$\mathrm{A}_{\mathrm{e}}$ (dashed curves) particles with

$d=0.2$ (blue), 0.4 (red), and

$1.0 \AA$ (black)

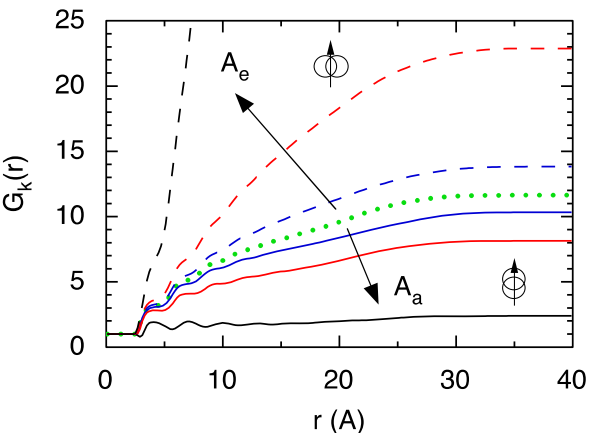

case $\left(A_{a}\right)$ as compared to the reference fluid (Ref). Nevertheless, the two angular probability functions monitoring preferential orientation averaged over the first coordination shell are for these fluids with different types of particles relatively close to each other.

Figure 3 displays the distance-dependent Kirkwood function $G_{\mathrm{k}}(r)$ for fluids containing reference particles and the two types of shape-asymmetric particles with $d=0.2,0.4$, and $1.0 \AA$. At small $r(r<2.5 \AA), G_{\mathrm{k}}(r)=1$, which follows from the definition (13) and that no neighbors are found at such short separations (Fig. 2(a)). At larger $r, G_{\mathrm{k}}(r)$ increases with appearance of some oscillations; the locations of shoulders and maxima are in accord with the location of maxima of the radial distribution functions; hence, these features in $G_{\mathrm{k}}(r)$ are linked to the uneven radial distribution of particles. The steady rise of $G_{\mathrm{k}}(r)$, however, indicates a net preference of parallel dipole orientation at all $r$. At separations, $r \approx L / 2, G_{\mathrm{k}}(r)$ approaches a limiting value. Noticeably, is that the limiting values of $G_{\mathrm{k}}(r)$ (and hence the dielectric permittivity) are strongly dependent on the type and the degree of particle shapeasymmetry. In the case of parallel orientation of the particle elongation and the dipole, the limiting value of $G_{\mathrm{k}}(r)$ decreases with increasing particle elongation, whereas for a perpendicular orientation of the particle elongation and the dipole the liming value of $G_{\mathrm{k}}(r)$ increases with increasing particle elongation. For example, whereas $\lim _{r \rightarrow \infty} G_{\mathrm{k}}(r) \approx 12$ for the reference fluid, the limit decreases to $\approx 8$ for a parallel and increases to $\approx 28$ for a perpendicular arrangement of the dipole and the particle elongation at $d=0.4 \AA$ (see also Table 2). The effects for particles with $d=1.0 \AA$ are even larger, here $\lim _{r \rightarrow \infty} G_{\mathrm{k}}(r) \approx 2$ for a parallel and $\approx 900$ for a perpendicular arrangement. In the latter case, the angular probability functions become oscillatory (data not shown), hence we have evidences of a spatially disordered and orientationally ordered fluid, hence a nematic phase. We may thus conclude that a deviation of the short-range interaction from spherical symmetry affects the distancedependent Kirkwood function (Fig. 3) stronger than the radial distribution (Fig. 2(a)) and angular probability (Fig. 2(b)) functions.

The sensitivity of the long-range nematic ordering on the short-range interaction as quantified by the distance-dependent Kirkwood function is supported by the results of the dipole domain analysis. Figure 4(a) displays the normalized probability function $P\left(G_{\mathrm{k}}^{\max }\right)$ and Fig. 4(b) the normalized probability function $P\left(R_{\max }\right)$ for fluids of the reference particle and the two types of size-asymmetric particles with $d=0.4 \AA$. We recall that $G_{\mathrm{k}}^{\text {max }}$ is a measure of the dipole alignment in a domain with radius $R_{\max }$. These probabilities show that $G_{\mathrm{k}}^{\max }$ increases approximately 2 -fold and $R_{\max }$ by ca. $30 \%$ for each step in the series $\mathrm{A}_{\mathrm{a}}$, Ref, and $\mathrm{A}_{\mathrm{e}}$. Furthermore, Fig. 4(c) displays the appearance of a large positive correlation between the values of $G_{\mathrm{k}}^{\max }$ and $R_{\max }$; a large domain size is accompanied by a large net dipole moment in the direction of the dipole of the central particle. Corresponding 
Table 2 Kirkwood $g_{\mathrm{K}}$-factor, reduced potential energy $U / N k T$, and reduced pressure $P V / N k T$ for reference and shape-asymmetric particles ${ }^{\mathrm{a}}$

\begin{tabular}{llccc}
\hline System & $d(\AA)$ & $g_{\mathrm{K}}{ }^{\mathrm{b}}$ & $U / N k T$ & $P V / N k T$ \\
\hline Ref & - & $11.7 \pm 0.8$ & $-8.501 \pm 0.002$ & $2.427 \pm 0.004$ \\
$\mathrm{~A}_{\mathrm{a}}$ & 0.1 & $12.1 \pm 0.8$ & $-8.443 \pm 0.002$ & $2.590 \pm 0.007$ \\
& 0.2 & $10.6 \pm 0.5$ & $-8.277 \pm 0.001$ & $3.074 \pm 0.005$ \\
& 0.4 & $8.3 \pm 0.5$ & $-7.680 \pm 0.001$ & $5.053 \pm 0.005$ \\
& 1.0 & $2.3 \pm 0.2$ & $-4.785 \pm 0.002$ & $18.51 \pm 0.01$ \\
$\mathrm{~A}_{\mathrm{e}}$ & 0.1 & $14.8 \pm 1.0$ & $-8.482 \pm 0.001$ & $2.564 \pm 0.005$ \\
& 0.2 & $16.6 \pm 2.0$ & $-8.405 \pm 0.001$ & $2.992 \pm 0.003$ \\
& 0.4 & $28.5 \pm 3.0$ & $-8.133 \pm 0.001$ & $4.693 \pm 0.004$ \\
& 1.0 & $880 \pm 20$ & $-6.672 \pm 0.003$ & $16.53 \pm 0.01$ \\
\hline
\end{tabular}

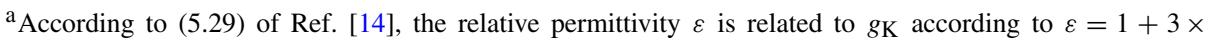
$\left[(4 \pi / 9)\left(\rho \mu^{2} / 4 \pi \varepsilon_{0} k T\right)\right] g_{\mathrm{K}}=1+3\left[(4 \pi / 9)\left(\rho^{*} \mu^{*^{2}} / T^{*}\right)\right] g_{\mathrm{K}}$. With the reduced variables inserted, the relation becomes $\varepsilon=1+10.2 g_{\mathrm{K}}$

$\mathrm{b}_{g_{\mathrm{K}}}=G_{\mathrm{K}}(r=\sqrt{3}(L / 2))$ with $L=42.7 \AA$

correlation plot of the data for the $\mathrm{A}_{\mathrm{e}}$ system with the largest value of $d, d=1.0 \AA$, displays a distribution centered at $R_{\max } \approx 37 \AA$ ( $\approx$ half the diagonal of the simulation box) and $G_{\mathrm{k}}^{\max } \approx 1500$ with a very limited broadening, implying that most of the 3000 particles form a single domain, being consistent with the large value of $\lim _{r \rightarrow \infty} G_{\mathrm{k}}(r)$.

We now continue by considering the $2 \mathrm{~d}$ radial-angular distribution function $g(r, \cos \theta)$, which is analog to the radial distribution function $g(r)$, though the $r$-dependence is made separately for different values of the angle $\theta$ with respect to the dipole of the central particle (see (9) and insert of Fig. 2(b)). Figure 5 displays contour plots of $g(r, \cos \theta)$ of fluids containing the reference particle and the two types of shape-asymmetric particles with $d=0.4 \AA$. As for the fluid containing particles with the spherical short-range interaction, Fig. 5(a) clearly shows that the radial distribution function is dependent on $\theta$. For $|\cos \theta|=1$, i.e., in the direction parallel or antiparallel to the dipole, $g(r, \cos \theta)$ displays two equal and global maxima of $\approx 3.2$ at $r=2.9 \AA$, whereas for $|\cos \theta|=0$, i.e., in the direction perpendicular to the dipole, the first maximum becomes $\approx 2.5$ and appears at $r=3.1 \AA$. Figure 5(a) also displays that the following minimum of $g(r, \cos \theta)$ appears at longer separation in the direction perpendicular than in the direction parallel to the dipole. Further analysis (data not shown) displays that these partly out-off phase oscillations of $g(r, \cos \theta)$ perpendicular and parallel to the direction of the dipole pertain at least three particle layers.

Figure 5(b) shows the corresponding contour plot for the shape-asymmetric particle with the size-elongation direction parallel $\left(\mathrm{A}_{\mathrm{a}}\right)$ to the dipole with $d=0.4 \AA$. Here, (i) the location of the first maximum of $g(r, \cos \theta)$ vs $r$ appears at $r \approx 3.1 \AA$ and is almost independent of $\cos \theta$ and (ii) the maximum has now a weaker dependence on $\cos \theta$ (varies between 2.7 and 3.1). Hence, in this case the shape asymmetry of the short-range interaction to a large degree counteracts the order arising from the electrostatic dipole-dipole interaction. Consistently, for this fluid we found a smaller limiting distance-dependent Kirkwood factor of 8 as compared to 12 for the reference fluid.

Finally, the corresponding contour plot for the shape-asymmetric particle with the sizeelongation direction perpendicular $\left(\mathrm{A}_{\mathrm{e}}\right)$ to the dipole is given in Fig. 5(c). Now we obtain an enhanced angular-dependent radial distribution function as compared to the reference fluid 
Fig. 4 (Color online)

(a) Normalized probability distribution $P\left(G_{\mathrm{k}}^{\max }\right)$,

(b) normalized probability distribution $P\left(R_{\max }\right)$, and (c) scatter plot of $G_{\mathrm{k}}^{\max }$ versus $R_{\text {max }}$ for dipolar domains of fluids containing reference particles (green) and $\mathrm{A}_{\mathrm{a}}$ (blue) and $\mathrm{A}_{\mathrm{e}}(\mathrm{red})$ particles with $d=0.4 \AA$
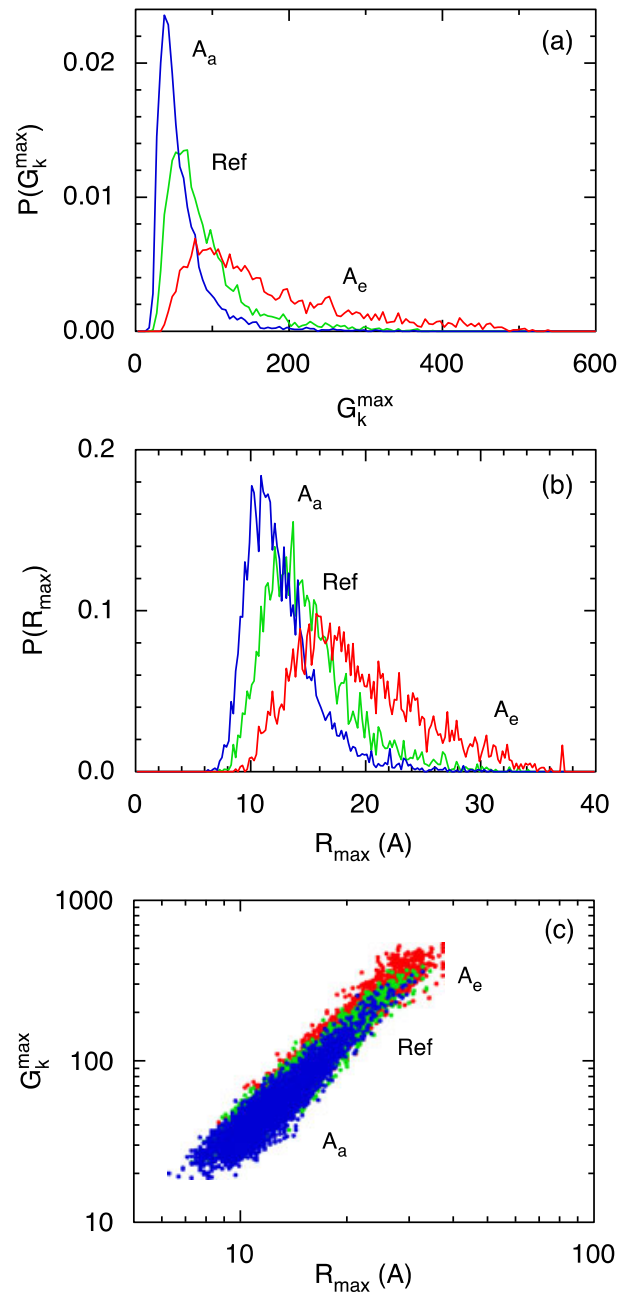

(cf. Figs. 5(c) and 5(a)), which possesses the largest maximal value 3.4 of the three fluids reported in Fig. 5. For this fluid, we found a limiting value for the distance-dependence Kirkwood factor of 28. As compared to the reference fluid, the fluid with $\mathrm{A}_{\mathrm{e}}$ particles with $d=0.4 \AA$ displays both a larger Kirkwood factor and larger dipole domains.

Hence, by suitable change of the short-range interaction among particles having an electrostatic dipole-dipole interaction, the non-isotropic density distribution of particles around a central particle may be altered such that orientational polarization of the fluid may either be (i) reduced or (ii) enhanced similar to what was found in paper I, though in paper I penalty functions were used to manipulate the radial distribution and angular orientation of the particles.

We have seen that the radially integrated and angular averaged dipole polarization typically increases from unity (Fig. 3). But what about the contributions to $G_{\mathrm{k}}(r)$ from particles located at different $\theta$ angles? Figure 6 provides the intrinsic local polarization $\mathfrak{p}(r, \cos \theta)$ as defined by (17) for the reference fluid and the two different types of shape-asymmetric particles with $d=0.4 \AA$. For the reference fluid, we see that $\mathfrak{p}$ amounts to $\approx 0.6$ in the 

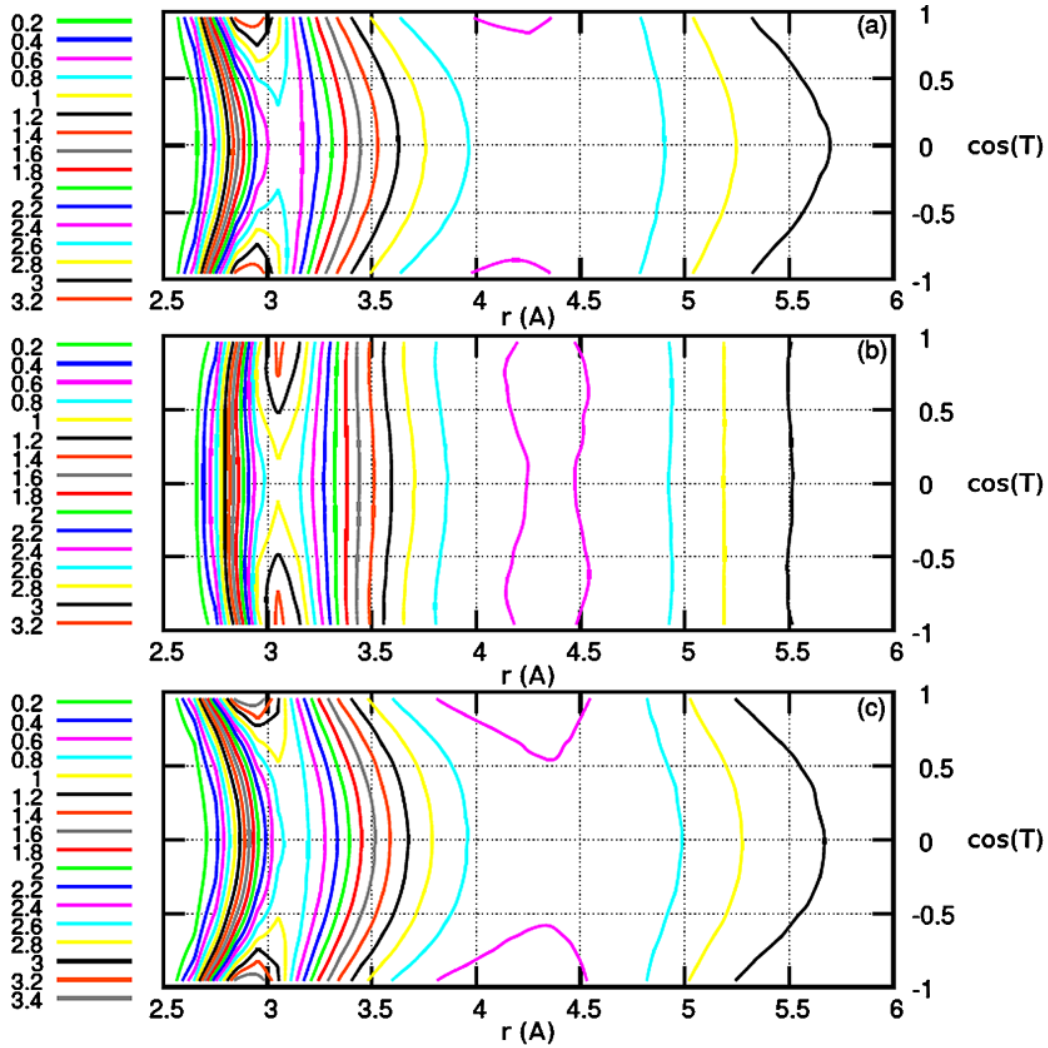

Fig. 5 (Color online) Two-dimensional radial-angular distribution function $g(r, \cos \theta)$ of fluids containing (a) reference particles and (b) $\mathrm{A}_{\mathrm{a}}$ and (c) $\mathrm{A}_{\mathrm{e}}$ particles with $d=0.4 \AA$

first coordination shell $(r \approx 2.9 \AA)$ in the directions parallel and antiparallel to the dipole vector, whereas it is between 0.0 and 0.1 in the direction perpendicular to the dipole vector (Fig. 6(a)). For very short separations $(r \approx 2.5-2.7 \AA)$, the largest intrinsic polarization becomes $\approx 0.7$ whereas it becomes slightly negative $\approx-0.1$ for orientations perpendicular to dipole axis. Moreover, at $r \approx 4.2 \AA$, corresponding to the first minimum of the radial distribution function, $\mathfrak{p}$ is between 0.0 and 0.1 at all angles and at $r \approx 5.9 \AA$, corresponding to the second maximum of the radial distribution function, between 0.0 and 0.2 . Thus, the intrinsic polarization near the dipolar particle is largest in the dipole direction, where also the strongest dipole-dipole interaction prevails. Beyond this region the intrinsic polarization is basically slightly positive and nearly uniform.

The corresponding $2 \mathrm{~d}$ representations of the intrinsic polarization for fluids containing particles with the shape-asymmetric short-range interactions with $d=0.4 \AA$ have also been examined. For the size-elongation parallel to the dipole, the region with negative polarization becomes more extended (Fig. 6(b)). We see that the volume with nearby pairs at $\theta=\pi / 2$ with negative polarization is enlarged and a large part of the volume between $r=4$ and $6 \AA$ A possess slightly negative polarization. On the contrary, for the size-elongation perpendicular to the dipole the positive polarization is enhanced and the enhancement appears to be rather uniformly distributed (Fig. 6(c)). Again the dipole polarization appears basically uniform, now at $\approx 0.1$, except around the global density maxima appearing at $|\cos \theta|=1$. 


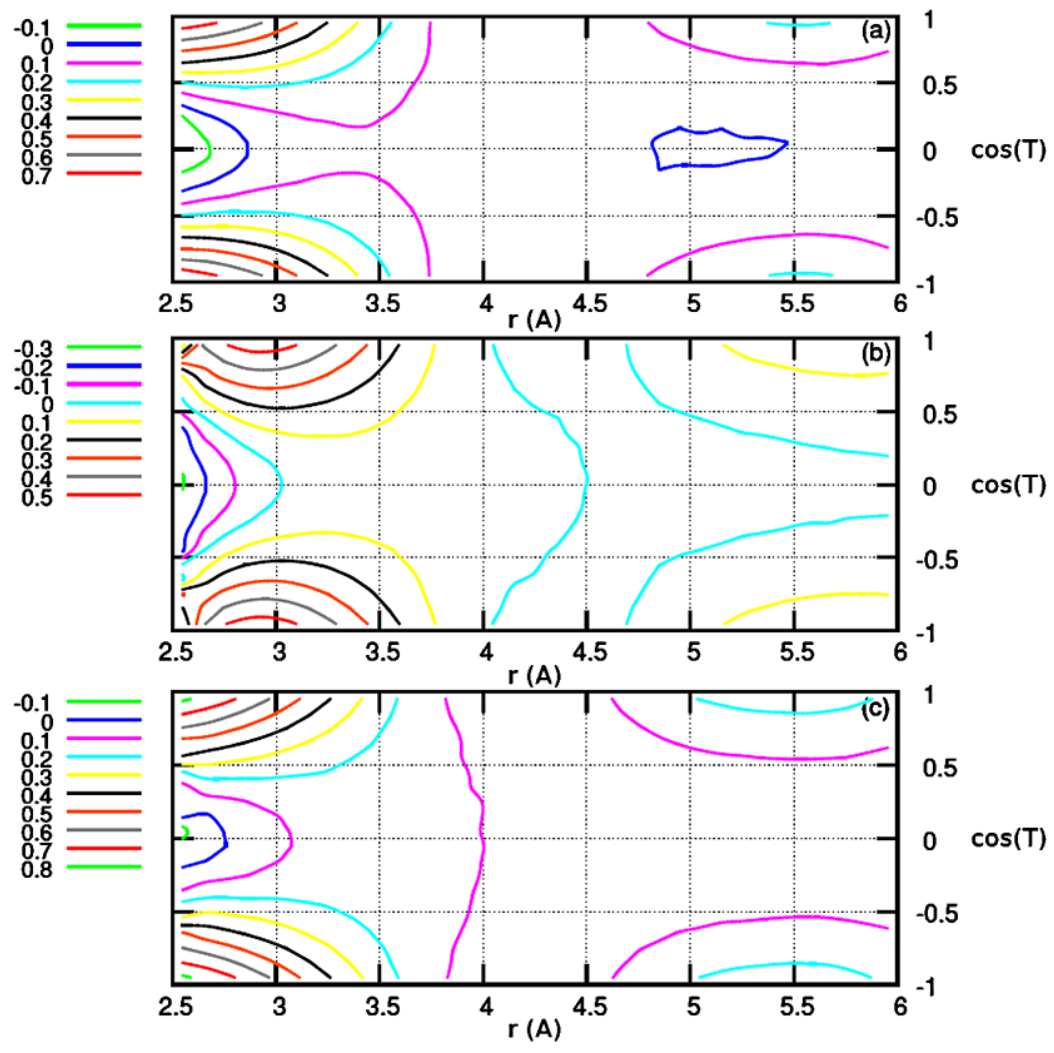

Fig. 6 (Color online) Two-dimensional intrinsic polarization function $\mathfrak{p}(r, \cos \theta)$ of fluids containing (a) reference particles and (b) $\mathrm{A}_{\mathrm{a}}$ and (c) $\mathrm{A}_{\mathrm{e}}$ particles with $d=0.4 \AA$

Fig. 7 Change of the two-dimensional intrinsic polarization function $\Delta \mathfrak{p}(d)=\langle\mathfrak{p}(r, \cos \theta ; d)-\mathfrak{p}(r, \cos \theta ; 0)\rangle$ averaged over $r$ and $\cos \theta$ for $\mathrm{A}_{\mathrm{a}}$ particles (negative $d$ ) and $\mathrm{A}_{\mathrm{e}}$ particles (positive $d$ ). The curve is a visual guide and the dashed part indicates the appearance of a discontinuity due to a phase transition to an orientational ordered (nematic) state at large $d$

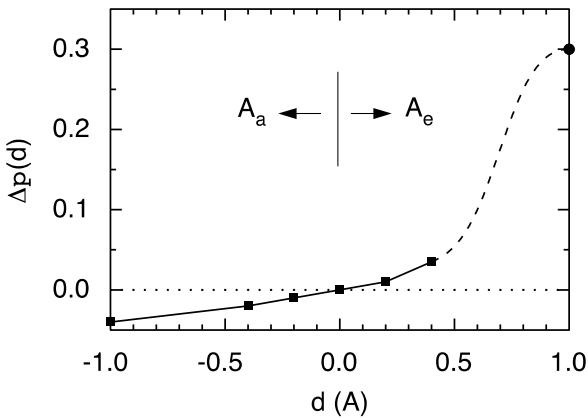

By making differential plots with respect to the reference fluid (data not shown), we find throughout that the change of the intrinsic polarization is fairly uniform across all the fluids with shape-asymmetric particles investigated. Figure 7 displays this change $\Delta \mathfrak{p}(d)=$ $\mathfrak{p}(d)-\mathfrak{p}(0)$ as function of $d$. At sufficiently small $|d|$, the response has to be linear in $d$, which is reasonable for $|d|$ up $0.4 \AA$. As to the fluid with $\mathrm{A}_{\mathrm{e}}$ particles with $d=1.0 \AA$, we have $\Delta \mathfrak{p} \approx 0.3$, and obviously this fluid has passed the phase boundary to a nematic phase. 
Table 3 Relative multipole-multipole interaction energies ${ }^{\mathrm{a}}$

\begin{tabular}{lllll}
\hline$\mu_{\mathrm{q}}(e \AA)$ & $u_{12} / u_{11}$ & $u_{22} / u_{11}$ & $u_{13} / u_{11}$ & $u_{33} / u_{11}$ \\
\hline 0.2 & -0.1 & -0.02 & - & - \\
0.5 & -0.3 & -0.1 & - & - \\
1.0 & -0.6 & -0.5 & 0.08 & 0.02 \\
2.0 & -1.2 & -1.8 & 0.2 & 0.06 \\
4.0 & - & - & 0.3 & 0.2 \\
8.0 & - & - & 0.6 & 1.0 \\
\hline
\end{tabular}

${ }^{\mathrm{a}}$ Evaluated from (A5) to (A9) with (i) $\delta=0.1 \AA$ and $r=3.0 \AA$ giving $(\delta / r) \approx 0.033$ and (ii) $\mu=0.34 e \AA$

Table 2 also provides the total potential energy, $U$, and the pressure, $P$, for reference and shape-asymmetric particles. The total potential energy becomes less negative at increasing shape asymmetry, independently of shape. For a given $d$, the change is largest for particle elongation parallel to the dipole direction. A split of $U$ into short-range and electrostatic contributions shows that (i) both of them become less negative for particle elongation parallel and (ii) the former becomes less negative and the latter more negative for particle elongation perpendicular to the dipole direction (data not shown). Thus, these data shows a co-variation between an increase of the electrostatic interaction and an increased Kirkwood factor. Furthermore, Table 2 displays that the pressure increases with increasing $d$ and the concomitant larger particle volume fraction, consistent with the notion that the contribution of the shortrange interaction to the pressure is important and strongly dependent on the particle volume fraction.

\subsection{Modified Electrostatic Interaction}

We will now consider fluids where the particles in addition to a dipole posses either a quadrupole or an octupole of various magnitudes. Hence, the electrostatic dipole-dipole interaction will gradually be challenged by increasing strength of electrostatic interactions that are (i) more short-ranged and (ii) has a stronger angular dependence. Of course, our aim is to examine how the presence of the higher-order electrostatic multipoles perturbs the dipole polarization of the fluid.

Ratios of relevant multipole-multipole interaction energies at appropriate particle separation will give a first order estimate of the dominating electrostatic interaction at that distance. As earlier mentioned, the leading quadrupole and octupole moments were built up by combining several dipoles $\boldsymbol{\mu}_{\mathrm{q}}$ of equal magnitude, see Fig. 1. For simplicity, the strength of the quadrupoles and octupoles will be given in terms of the magnitude $\mu_{\mathrm{q}}$. For particle separations corresponding to nearest-neighbor contact, (i) the quadrupole-quadrupole interaction is stronger than the dipole-dipole and quadrupole-dipole interactions at the largest $\mu_{\mathrm{q}}$ considered $\left(\mu_{\mathrm{q}}=2.0 e \AA\right)$, and (ii) the octupole-octupole interaction is equal to the dipoledipole interaction and stronger than the octupole-dipole interaction at the largest $\mu_{\mathrm{q}}$ considered ( $\mu_{\mathrm{q}}=8.0 e \AA$ ) (see Table 3). On the basis of these findings and as the structural observations could be rationalized without invoking the quadrupole-dipole and octupoledipole interactions, they are omitted in the further discussion.

\subsubsection{Quadrupoles}

Figure 8 displays the distance-dependent Kirkwood function for fluids that contain reference particles and particles with either of two types of quadrupoles of various strengths beside the 
Fig. 8 (Color online)

Distance-dependent Kirkwood function $G_{\mathrm{k}}(r)$ of fluids containing reference particles (green) and $\mathrm{Q}_{\mathrm{a}}$ (solid curves) and $\mathrm{Q}_{\mathrm{e}}$ (dashed curves) particles with $\mu_{\mathrm{q}}=0.2$ (blue), 0.5 (red), 1.0 (black), and $2.0 \mathrm{e} \AA$ (purple)

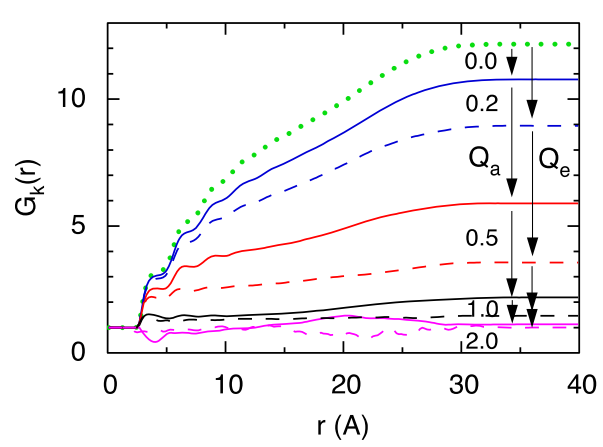

Fig. 9 (Color online) Scatter plot of $G_{\mathrm{k}}^{\max }$ versus $R_{\max }$ for dipolar domains of fluids containing reference particles (green) and (a) $\mathrm{Q}_{\mathrm{a}}$ and (b) $\mathrm{Q}_{\mathrm{e}}$ particles with $\mu_{\mathrm{q}}=0.2$ (green), 0.5 (blue), 1.0 (red), and $2.0 e \AA$
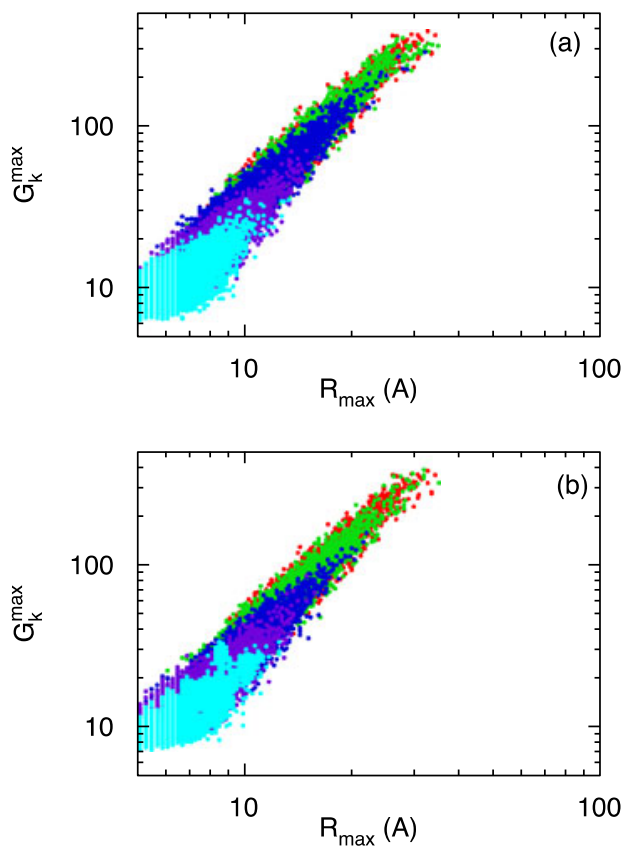

dipole. Starting with the fluid containing the reference particles that possess only a dipole, we see that (i) the distance-dependent Kirkwood function decreases gradually at increasing quadrupole moment to about $G_{\mathrm{k}}(r) \approx 1$ for $\mu_{\mathrm{q}}=1.0 e \AA$ and remains basically the same for the largest quadrupole moments obtained for $\mu_{\mathrm{q}}=2.0 \mathrm{e} \AA$ and (ii) the decrease is faster for the $\mathrm{Q}_{\mathrm{e}}$ series as compared to the $\mathrm{Q}_{\mathrm{a}}$ one. Hence, these results signify that for both the axial and equatorial quadrupoles used, the addition of either of them to a dipole weakens the structure driven by the dipole-dipole interaction, and we will in the following examine that in somewhat more detail.

The scatter plot of $G_{\mathrm{k}}^{\max }$ versus $R_{\max }$ for dipolar domains of these fluids is displayed in Fig. 9. Starting with the reference fluid, where the lower limits are $G_{\mathrm{k}}^{\max } \approx 30$ and $R_{\max } \approx 8 \AA$ (see Fig. 4), we find that the magnitude of the dipole moment of the domains and the size of the domains decrease regularly at increasing strength of the quadrupole moment. This holds both for axial (Fig. 9(a)) and equatorial (Fig. 9(b)) quadrupoles and the decrease is qualitatively similar. 


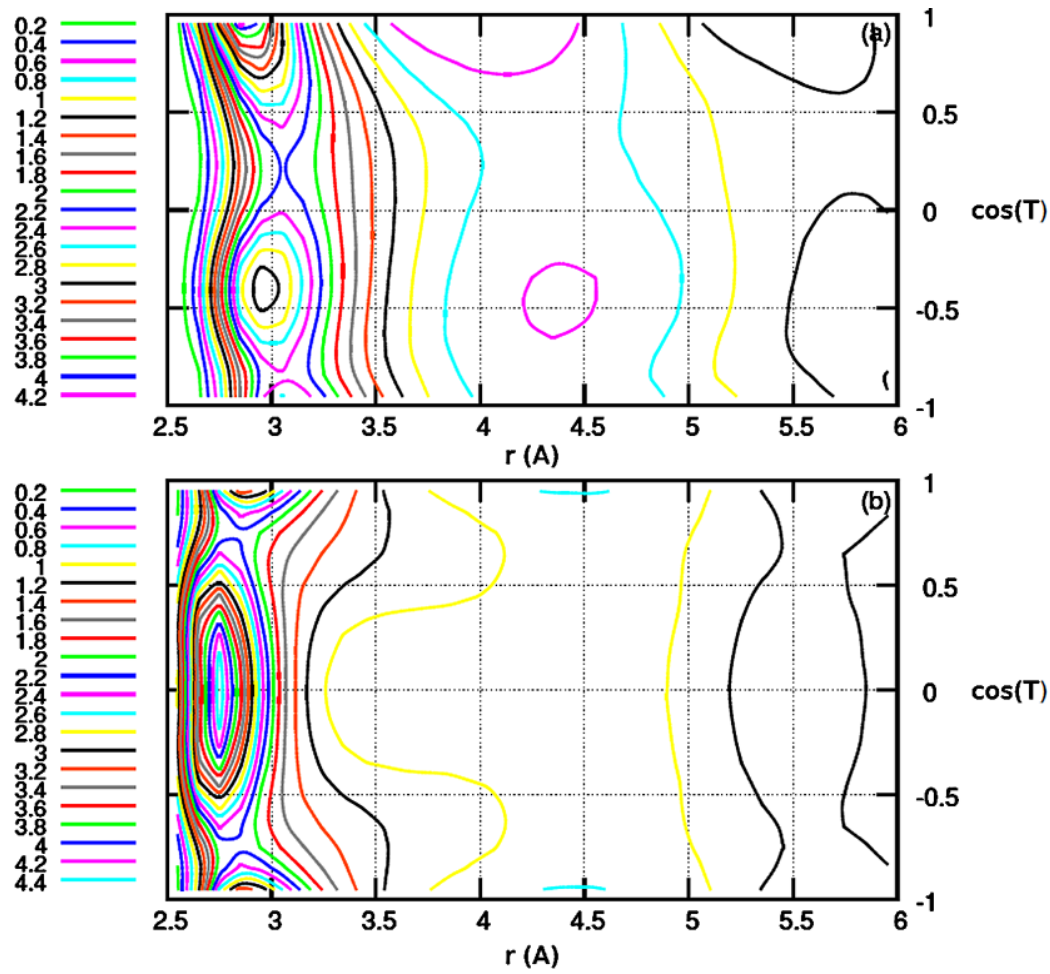

Fig. 10 (Color online) Two-dimensional radial-angular distribution function $g(r, \cos \theta)$ of fluids containing (a) $\mathrm{Q}_{\mathrm{a}}$ and (b) $\mathrm{Q}_{\mathrm{e}}$ particles with $\mu_{\mathrm{q}}=1.0 e \AA$

Nevertheless, the two-dimensional radial-angular distribution functions displayed in Fig. 10 at $\mu_{\mathrm{q}}=2.0 e \AA$ shows that the short-range structure differ. For particles with an axial quadrupole, local maxima appear at separation $r \approx 3.0 \AA$ at $\cos \theta=1$ and -0.4 (Fig. 10(a)). Jointly, these maxima imply that nearest-neighboring particles possess a preference for their symmetry axes to be nearly perpendicular to each other ( $T$-shaped structure). This local structure differs from that found in the reference fluid, but is close to that prevalent in fluids of particles possessing only axial quadrupoles. Furthermore, the lack of symmetry with respect to $\cos \theta=0$ originates from the fact that the dipole and quadrupole have odd $(l=1)$ and even $(l=2)$, respectively, parity with respect to reflection in the molecular $x y$-plane (see also Fig. 1).

For the equatorial quadrupole, the preferred short-range structure involves the two symmetry axes localized parallel (side-by-side structure), an arrangement that maximizes the quadrupole-quadrupole attraction. As the quadrupole-quadrupole interaction is unaffected whether the symmetry axes are parallel or antiparallel, the later prevails due to the dipoledipole interaction. At $\mu_{\mathrm{q}}=2.0 \mathrm{e \AA}$, the side-by-side arrangement dominates the shortrange structure in the fluid (Fig. 10(b)). The stronger $r$-dependence of the quadrupolequadrupole interaction as compared to the dipole-dipole interaction shifts the maximum of $g(r, \cos \theta \approx 0$ ) from $r \approx 2.9 \AA$ (Fig. 5(a)) to $r \approx 2.75 \AA$. The local maxima at $r \approx 2.9 \AA$ and $|\cos \theta|=1$ are traces of the dipole-dipole interaction.

To conclude, the introduction of a quadrupole in addition to the dipole gives rise to short-range structures that are incompatible with that of particles with only a dipole. This 
Fig. 11 (Color online)

Distance-dependent Kirkwood

function $G_{\mathrm{k}}(r)$ of fluids

containing reference particles

(green) and $\mathrm{O}_{+}$(solid curves)

and $\mathrm{O}_{-}$(dashed curves) particles

with $\mu_{\mathrm{q}}=1.0$ (blue), 2.0 (red),

4.0 (black), and $8.0 e \AA$ (purple)

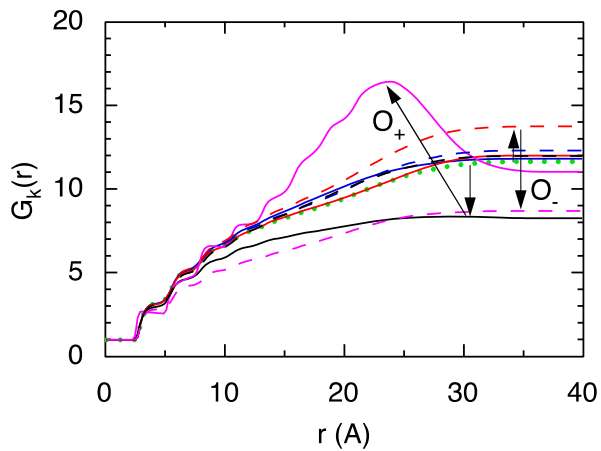

fact lies behind the gradually reduction of the dipole polarization at increasing quadrupole strength.

\subsubsection{Octupoles}

We recall that the two types of particles with octupoles considered possess axial octupoles. The directions of the symmetry axes of the axial octupole and of the dipole are parallel for the $\mathrm{O}_{+}$particles and antiparallel for the $\mathrm{O}_{-}$particles.

The corresponding distance-dependent Kirkwood function for fluids containing reference particles and particles with modified electrostatic interaction involving octupoles obtained with $\mu_{\mathrm{q}}=1.0,2.0,4.0$, and $8.0 \mathrm{e \AA}$ beside the dipole $\boldsymbol{\mu}$ are given in Fig. 11. First, we see that the changes of $G_{\mathrm{k}}(r)$ are generally smaller in the presence of octupoles as compared to the presence of quadrupoles. Second, the influence of the octupole moment of the $\mathrm{O}_{+}$ and $\mathrm{O}_{-}$particles differs. With $\mathrm{O}_{+}$particles, at $\mu_{\mathrm{q}}=4.0 e \AA G_{\mathrm{k}}(r)$ has decreased, but at the largest $\mu_{\mathrm{q}}$ considered $G_{\mathrm{k}}(r)$ has increased again and now displays a prominent maximum at $r \approx 22 \AA$. With $\mathrm{O}_{-}$particles, $G_{\mathrm{k}}(r)$ increases slightly at $\mu_{\mathrm{q}}=4.0 e \AA$, but has reduced considerably at $\mu_{\mathrm{q}}=8.0 \AA$.

The corresponding scatter plots of $G_{\mathrm{k}}^{\max }$ versus $R_{\max }$ for dipolar domains are displayed in Fig. 12. As to the $\mathrm{O}_{+}$fluid (Fig. 12(a)), the fluctuations of $G_{\mathrm{k}}^{\max }$ and $R_{\max }$ for the two smaller values of $\mu_{\mathrm{q}}$ are similar to that of the reference fluid. However, with $\mu_{\mathrm{q}}=4.0 \mathrm{e} \AA$ the larger dipolar domains appearing in the reference fluid are suppressed, consistent with smaller amplitude of the distance-dependent Kirkwood function. With $\mu_{\mathrm{q}}=8.0 \mathrm{e} \AA$, in addition to the suppression of the larger domains, also the smaller dipolar domains are suppressed, consistent with the now increased amplitude of the distance-dependent Kirkwood function. Regarding the $\mathrm{O}_{-}$fluid (Fig. 12(b)), the change of values of $G_{\mathrm{k}}^{\max }$ and $R_{\max }$ as well as their correlation remains small except with $\mu_{\mathrm{q}}=8.0 \mathrm{e} \AA$, at which the larger dipolar domains are suppressed.

As for particles with quadrupoles, the two-dimensional radial-angular distribution function displayed in Fig. 13 at $\mu_{\mathrm{q}}=8.0 e \AA$ shows that the short-range structure differ between the two fluids with octupoles differently oriented with respect to the dipole. With coinciding and parallel dipole and octupole symmetry axes, (i) two equivalent and global maxima appear at $r \approx 2.7 \AA$ and $|\cos \theta|=1$, (ii) two equal and local maxima occur at $r \approx 3.1 \AA$ and $|\cos \theta|=0.35$, and (iii) two equal and local maxima at $r \approx 5.4 \AA$ and $|\cos \theta|=1$ are found (Fig. 13(a)). Maxima (i) are related to the similar maxima at $r \approx 2.9 \AA$ and $|\cos \theta|=1$ of the reference fluid. Here these two maxima are much more prominent, more localized in the $(r, \theta)$-space, and appear at shorter separation, all as a consequence of the synergistic effect 
Fig. 12 (Color online) Scatter plot of $G_{\mathrm{k}}^{\max }$ versus $R_{\max }$ for dipolar domains of fluids containing reference particles (green) and (a) $\mathrm{O}_{+}$and (b) $\mathrm{O}_{-}$ particles with $\mu_{\mathrm{q}}=1.0$ (green), 2.0 (blue), 4.0 (red), and $8.0 e \AA$ (light blue)
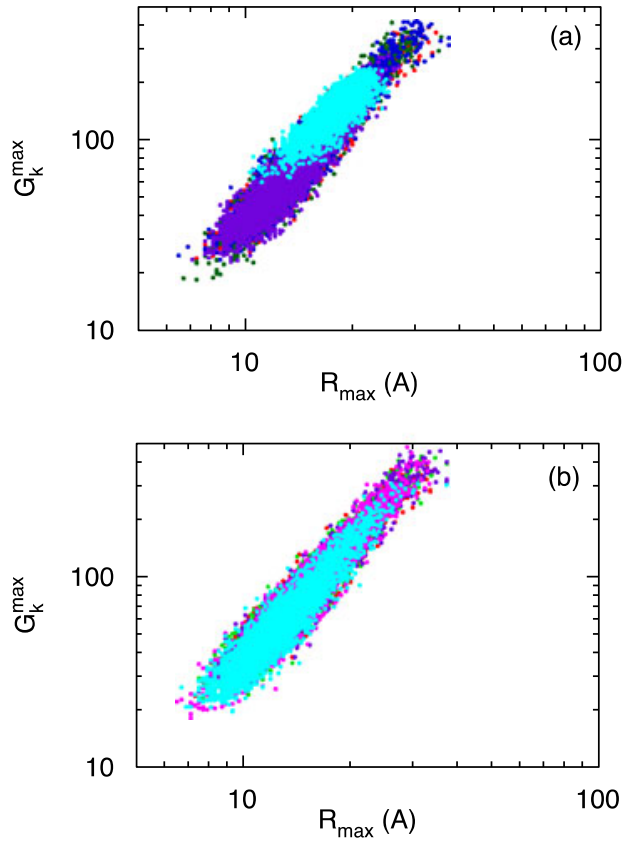

of the parallel octupole and dipole symmetry axes. Maxima (ii) appear from the angulardependence of the octupole-octupole interaction Finally, the appearance of maxima in the second nearest-neighbor only at $|\cos \theta|=1$ but not at $|\cos \theta|=0.35$ is consistent with the much stronger distance-dependence of the octupole-octupole interaction as compared to the distance-dependence of the dipole-dipole interaction, cf. (A.7) and (A.5) of Appendix.

For antiparallel octupole and dipole symmetry axes, only two major maxima remain (Fig. 13(b)). The maxima at $|\cos \theta| \approx 1$ of the reference system have vanished due to the (partly) compensating effects of the octupole and dipole in this volume. Only the two other maxima arising from the octupole-octupole interaction, albeit slightly shifted with respect to the poles, remain.

\section{Discussion}

\subsection{On the Origin of the Dipole Polarization}

For the reference particles composed of a LJ site with an embedded ideal dipole, the angulardependent interaction stems solely from the electrostatic dipole-dipole interaction (6). For example, given the separation $r$ between two dipoles, their maximal attractive interaction occurring at $|\cos \theta|=1$ is twice as large as their maximal attraction at $\cos \theta=0$ [follows directly from (6)]. As a consequence, (i) the radial density distribution becomes angular dependent with global maxima occurring at $|\cos \theta|=1$ and $r=2.9 \AA$ and the largest equatorial ( $\cos \theta=0$ ) density occurring at $r=3.1 \AA$ (see Fig. 5(a)) and (ii) the intrinsic polarization is strongly positive where the global density maxima occur and near zero equatorially.

On the basis of these results, and as also those discussed in Paper I, we suggest that the built-up of a large positive distance-dependent Kirkwood function, as displayed in Fig. 3, is characterized by four issues. These are illustrated in Fig. 14 and are: (a) a preferential 


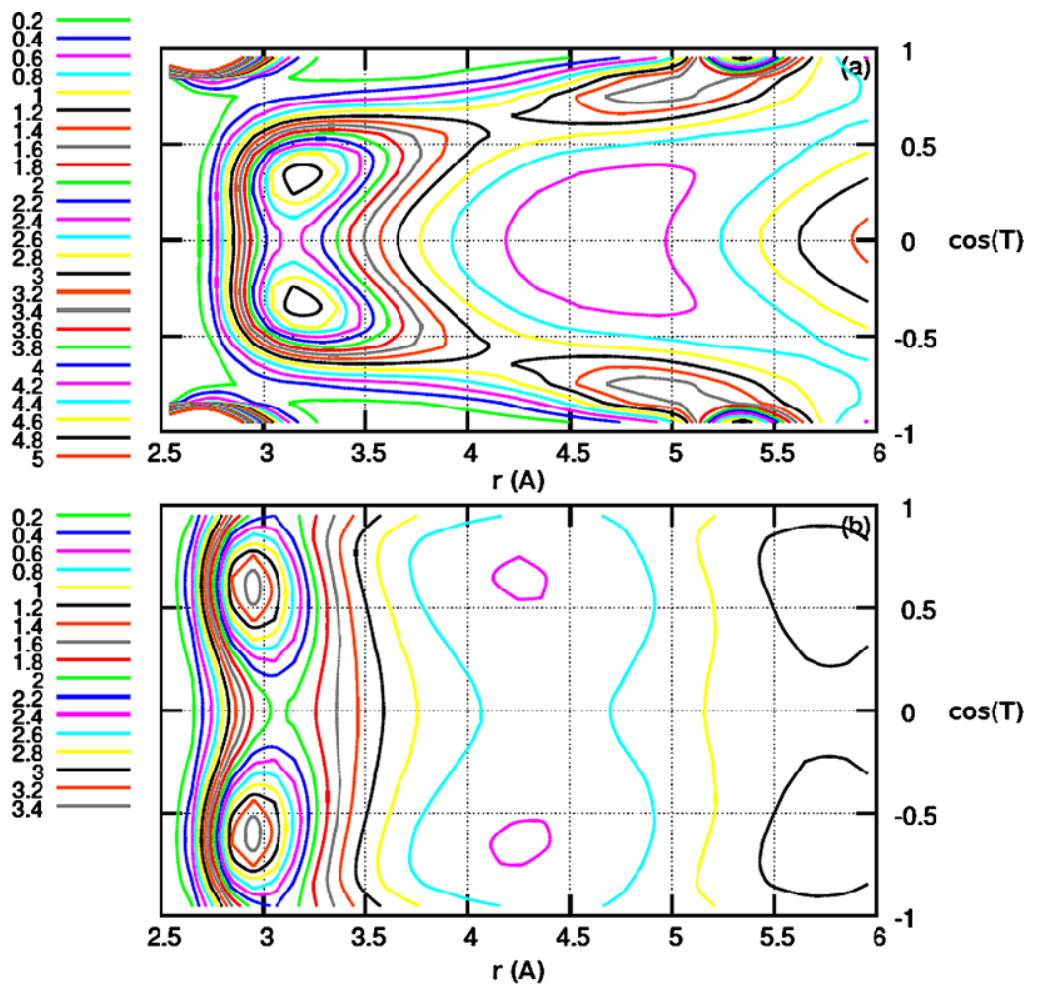

Fig. 13 (Color online) Two-dimensional radial-angular distribution function $g(r, \cos \theta)$ of fluids containing (a) $\mathrm{O}_{+}$and (b) $\mathrm{O}_{-}$particles with $\mu_{\mathrm{q}}=8.0 \mathrm{e} \AA$. In (a), the highest iso- $g$ curve is at 5.0, truncating the representation of the maxima at $r=2.7 \AA$ and $|\cos \theta|=1$

localization of neighboring particles to $|\cos \theta|=1$, (b) particles at $|\cos \theta| \approx 1$ are located closer to the central particle as compared to the equatorial ones, (c) particles at $|\cos \theta| \approx 1$ give rise to a large positive contribution to $G_{\mathrm{k}}(r)$ as the dipole-dipole interaction at this separation exceeds the thermal energy, and $(d)$ the dipole polarization of equatorially neighboring particles is nearly zero instead of the expected antiparallel orientation, which should have given a negative contribution to $G_{\mathrm{k}}(r)$. We attribute the absence of antiparallel orientation of equatorially located dipoles to be an effect of the ordering of the nearby dipoles at $|\cos \theta|=1$, as the field of those dipoles and of the central dipole to a great extent cancel each other. This cancellation is a consequence of the spatial dependence of the dipole-dipole interaction and of the packing pattern of spheres.

In short, the long-range dipolar polarization of a medium originates from a combination of the short-range packing and the more long-ranged dependent dipole-dipole potential of the particles as was found in paper I.

\subsection{Effect of Molecular Shape}

When the shape of the interacting particles is changed, their short-range packing is affected. In the case of the $A_{a}$ particles, they are elongated in the dipole direction. A comparison of Figs. 5(a) and 5(b) showed that the angular preference is weakened (issue a) and the difference of the location of the first radial maximum is absent (issue b). Furthermore, a comparison of Figs. 6(a) and 6(b) shows that the positive polarization in volumes at $|\cos \theta| \approx 1$ is 
Fig. 14 (Color online)

Illustration of some features of nearest-neighbor spatial and angular correlation of neighboring dipoles (labeled 2-4) to dipole (1) related to a large dipole polarization: (a) angular density dependence,

(b) angular-dependent separation-dependence,

(c) dipole-dipole correlation for axial located neighbors ( 2 and $\mathbf{3}$ ), (d) essentially zero dipole-dipole correlation for an equatorial located neighbor (4)
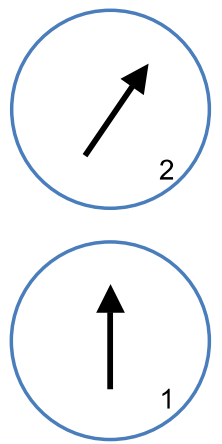

(a)
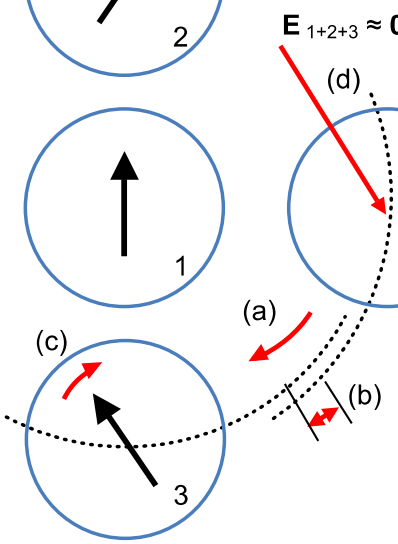

(d)

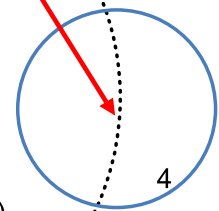

(b)

reduced (issue c) and the polarization of equatorially located dipoles is slightly negatively shifted (issue d). All these (coupled) aspects reduce the dipole polarization of the fluid composed of the $A_{a}$ particles as seen in Figs. 3 and 4. Our observed reduced dipolar order at increasing elongation parallel to the dipole axis is in agreement with reduced dielectric permittivity found by Morriss for similar, but non-idential systems [8]. In the case with the $A_{e}$ particles, where the elongation is perpendicular to the dipole direction, similar but opposite effects occur, including the appearance of a nematic dipolar ordered phase at large particle elongation.

Thus, at the current dipole-dipole interaction strength, only a weak shape change (aspect ratio $<1.1$ ) was required to cover the full range from a very small dipole polarization to a nematic dipolar order. At decreasing dipole-dipole interaction strength, stronger shapevariation is expected to be required to achieve the same response.

\subsection{Effect of Higher Order Electrostatic Multipole Moments}

The addition of higher order electrostatic interactions also affected the dipole polarization. In the case of addition of quadrupoles, Fig. 10(a) showed that a T-like configuration of the symmetry axes of neighboring particles is dominating for sufficiently large axial quadrupoles, and Fig. 10(b) displayed a side-by-side conformation for equatorial quadrupoles (as appearing in corresponding fluids of particles with quadrupole moments and with a quadrupolequadrupole interaction larger then the thermal energy). As the strength of the quadrupolequadrupole interaction becomes comparable to the dipole-dipole interaction for neighboring particles, the quadrupole-quadrupole interaction breaks down the dipole polarization as seen in Figs. 8 and 9. This appears both for the axial and the equatorial quadrupole.

Patey and Valleau [3] investigated a hard-sphere fluid at the reduced density $\rho^{*} \equiv \rho \sigma^{3}=$ 0.8344 with $\sigma$ now denoting the hard-sphere diameter, where the particles possessed a reduced dipole moment $\mu^{*} \equiv \mu /\left(4 \pi \varepsilon_{0} k T \sigma^{3}\right)^{1 / 2}=1$ at variable axial quadrupoles parallel to the dipole. The initial dipolar hard-sphere fluid possessed a dielectric permittivity $\varepsilon=7.7 \pm 1.3$ and displayed a reduction at increasing quadrupole moment. The largest slope of $\mathrm{d} \varepsilon / \mathrm{d} Q^{*}$ appeared at $Q^{*} \equiv Q /\left(4 \pi \varepsilon_{0} k T \sigma^{5}\right)^{1 / 2}=1$ and $\varepsilon=2.3 \pm 0.5$ was achieved at large $Q^{*}$-a result very much in line with ours given in Fig. 8. 
Fig. 15 Distance-dependent Kirkwood function $G_{\mathrm{k}}(r)$ at $r=25 \AA$ vs $\mu_{\mathrm{q}}$ of fluids containing $\mathrm{O}_{+}$(positive $\mu_{\mathrm{q}}$ ) and $\mathrm{O}_{-}$(negative $\mu_{\mathrm{q}}$ ) particles. Data from a few simulations with other values of $\mu_{\mathrm{q}}$ than given in Table 3 are also included

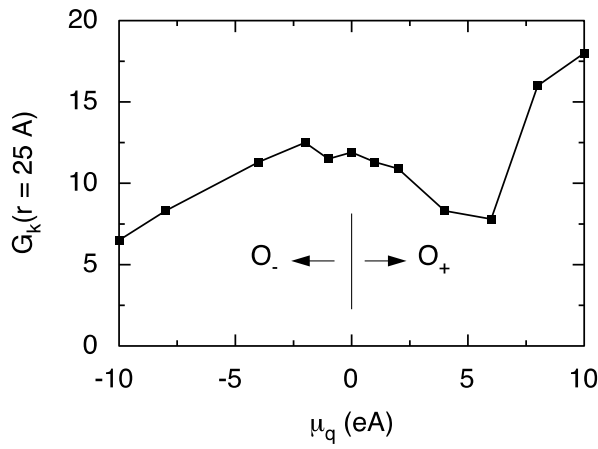

The analysis of the effect of adding an axial octupole is more complex and interesting. We note that for small octupoles, their effect can be regarded as a perturbation and hence we expect a linear response about the octupole moment $O_{\mathrm{zzz}}=0$. Figure 15 shows the distance-dependent Kirkwood function evaluated at $r=25 \AA$ as a function of $\mu_{\mathrm{q}}$ with here $Q_{\mathrm{zzz}}=Q_{30}=12 \mu_{\mathrm{q}} \delta^{2}$ and $\delta$ constant. Indeed, within the statistical uncertainty, the data in Fig. 15 displays a linear responds to a perturbation for $\left|\mu_{\mathrm{q}}\right|<2 e \AA$. An analysis of $g(r, \cos q)$ reveals that positive values of $\mu_{\mathrm{q}}$ induce a higher degree of structuring of orientationally ordered regions, but $\mathfrak{p}$ shows that regions with a significant contribution to the dipole polarization decrease. However, these two effects almost balance each other, and the total effect is that a positive $O_{\mathrm{zzz}}$ induces a small reduction of the dipole polarization and negative $O_{\mathrm{zzz}}$ a small increase. The increased structuring for larger and positive $O_{\mathrm{zzz}}$ is consistent with that the absolute minimum in the dipole-dipole interaction is reinforced by the added octupole. For example, this is shown by a decreased shortest separation between such pair of particles for positive $O_{\mathrm{zzz}}$ leading to a splited first peak of the radial distribution function (data not shown but could be inferred from Fig. 13(a)).

For larger perturbations, the system enters into another regime where the octupoleoctupole interactions strongly influences the structure. For large and positive values of $O_{\mathrm{zzz}}$, we note that the structure was continuously enforced, and was associated with a decrease in the dipole polarization at short distances and an increased of polarization at longer distances. We noted also that the radial distribution function becomes strongly perturbed. The simplest interpretation is an increased structuring on the molecular length scale making it possible for the enhanced polarization mechanism acting to operate also on longer distances. One view is that at the largest values of $O_{\mathrm{zzz}}$ a highly ordered long-range structure is formed, dominated by the octupole-octupole interaction. This structure favors large polarization. The domain analysis in Fig. 9(b) revealed one more interesting detail. Upon increasing $O_{\mathrm{zzz}}$, first the probability of larger domains is reduced, whereas later a decrease of the probability of small domains appears. As a consequence, the size and dipole polarization of the domains become more homogeneous at $\mu_{\mathrm{q}}=8.0$ indicating a highly structured system. We do not yet know if the properties of these domains are determined by the size or the physics of the studied systems.

For negative $O_{\mathrm{zzz}}$, the enhanced dipole polarization starts to decrease and becomes small for highly negative values of $O_{\mathrm{zzz}}$. The major cause for this behavior is the destruction of the dipolar structure discussed above. For these larger values of $O_{\mathrm{zzz}}$, an antiparallel orientation of the symmetry axis of the two particles competes with the parallel one governed by the dipole-dipole interaction. The antiparallel orientation prevents the build up of a significant dipole polarization. The domain analyses for these systems were presented in Fig. 12(b), 
and it showed a systematic decrease of the domain sizes for large negative $O_{\mathrm{zzz}}$ consistent with the observed reduction of the dipole polarization.

Finally, a comparison with magnetic dipoles can be made. In the those systems, the local magnetic moments are aligned due to the electronic structure in the system; hence, the magnetic polarization is not driven by the dipolar interactions in the current system investigated here.

\section{Conclusions}

We have in this work shown that small changes of the short-range interaction may have far-reaching effect on the dipole polarization and hence on the dielectric permittivity. A decreased dipole polarization is achieved when the particle is extended in the dipolar direction and an increase polarization for an equatorial extension. Furthermore, we have rationalized the physics behind these observations, and thus establish an understanding of how to manipulate the properties of the interacting particles to obtain desired dipolar polarization. Beside molecular fluids, this should also be applicable to nano-sized polar particles and to particles with magnetic moment as electrostatic and magnet interactions have the same geometrical dependence. The opposite is also true, i.e., it is possible to manipulate the short-range packing of dipolar particles by the addition of a sufficiently strong electrical field.

The dipole polarization was also found to depend on the introduction of higher order electrostatic moments. Addition of axial or equatorial quadrupoles leads only to a decrease of the dipolar polarization, which was attributed to different reflection parity for a dipole and these quadrupoles. However, addition of axial octupoles could both reduce and enhance the dipole polarization depending on the relative strength of the multipole-multipole interactions and the relative orientation of the dipole and axial octupole of a particle.

Acknowledgements Financial support by the Swedish Research Council (VR) through the Linnaeus grant Organizing Molecular Matter (OMM) center of excellence (239-2009-6794) and through individual grants to PL (621-2007-5251) and GK (621-2007-5009) is gratefully acknowledged.

Open Access This article is distributed under the terms of the Creative Commons Attribution Noncommercial License which permits any noncommercial use, distribution, and reproduction in any medium, provided the original author(s) and source are credited.

\section{Appendix: Electrostatic Interaction Energy Between Two Electrostatic Multipoles}

The aim of this appendix is to (i) provide expressions of the interaction energies between two multipoles and (ii) in particular interaction energies involving dipoles, quadrupoles, and octupoles using relevant expressions of the quadrupole and octupole moments.

The electrostatic interaction energy between multipole $Q_{i}$ located at the origin and multipole $Q_{j}$ located at $\mathbf{R}=(R, \Omega)$ is given by

$$
\begin{aligned}
U= & \frac{1}{4 \pi \varepsilon_{0}} \sum_{l_{1}=0}^{\infty} \sum_{l_{2}=0}^{\infty} u_{l_{1} l_{2}} \\
u_{l_{1} l_{2}}= & \sum_{m_{1}=-l_{1}}^{l_{1}} \sum_{m_{2}=-l_{2}}^{l_{2}}(-1)^{l_{1}+M}\left[\frac{(2 L) !}{\left(2 l_{1}\right) !\left(2 l_{2}\right) !}\right]^{1 / 2} \sqrt{2 L+1}\left(\begin{array}{ccc}
l_{1} & l_{2} & L \\
m_{1} & m_{2} & -M
\end{array}\right) \\
& \times Q_{i, l_{1} m_{1}} Q_{j, l_{2} m_{2}} \frac{1}{R^{L+1}} C_{L M}^{*}(\Omega)
\end{aligned}
$$


where $u_{l_{1} l_{2}}$ denotes the partial interaction energy between moment $l_{1}$ of multipole $i$ and moment $l_{2}$ of multipole $j, Q_{l_{1} m_{1}}, l_{1}=(0,1, \ldots), m_{1}=\left(-l_{1},-l_{1}+1, \ldots, l_{1}-1, l_{1}\right)$ a multipole moment; (:::) a $3 j$ symbol; $C_{L M}(\Omega)$ Racah's unnormalized spherical harmonics; * complex conjugate; $L=l_{1}+l_{2}$ and $M=m_{1}+m_{2}$. With only dipoles present (i.e., $Q_{i, l_{1} m_{1}}=\mu_{i} \delta_{l_{1} 1} \neq 0$ and $\left.Q_{j, l_{20} m_{2}}=\mu_{j} \delta_{l_{2} 1} \neq 0\right),(6)$ is recovered.

We will consider three successive restrictions, viz.:

(i) symmetry axis of the axial multipole $i$ parallel to the $z$-axis, i.e., $Q_{i, l_{1} m_{1}}=Q_{i, l_{1} 0} \delta_{m_{1}, 0}$,

(ii) symmetry axis of the axial multipole $j$ parallel to the $z$-axis, i.e., $Q_{j, l_{2} m_{2}}=Q_{j, l_{2} 0} \delta_{m_{2}, 0}$, and

(iii) location of multipole $j$ on the $z$-axis, i.e., $C_{L M}(\Omega)=\delta_{M 0}$,

leading to simplified interaction energy expressions. With restriction (i), the interaction energy becomes

$$
\begin{aligned}
u_{l_{1} l_{2}}= & \sum_{m_{2}=-l_{2}}^{l_{2}}(-1)^{l_{1}+M}\left[\frac{(2 L) !}{\left(2 l_{1}\right) !\left(2 l_{2}\right) !}\right]^{1 / 2} \sqrt{2 L+1}\left(\begin{array}{ccc}
l_{1} & l_{2} & L \\
0 & m_{2} & -m_{2}
\end{array}\right) \\
& \times Q_{i, l_{1} 0} Q_{j, l_{2} m_{2}} \frac{1}{R^{L+1}} C_{L m_{2}}^{*}(\Omega)
\end{aligned}
$$

whereas with restrictions (i) and (ii), we obtain

$$
u_{l_{1} l_{2}}=(-1)^{l_{2}}\left(\frac{L !}{l_{1} ! l_{2} !}\right) Q_{i, l_{1} 0} Q_{j, l_{2} 0} \frac{1}{R^{L+1}} C_{L 0}(\Omega)
$$

and with restrictions (i)-(iii), we get

$$
u_{l_{1} l_{2}}=(-1)^{l_{2}}\left(\frac{L !}{l_{1} ! l_{2} !}\right) Q_{i, l_{1} 0} Q_{j, l_{2} 0} \frac{1}{R^{L+1}} .
$$

To make it simple, we will only consider the multipole-multipole interaction energies under restrictions (i)-(iii). In particular, with $Q_{10}=\mu, Q_{20}=4 \mu_{\mathrm{q}} \delta$, and $Q_{30}=12 \mu_{\mathrm{q}} \delta^{2}$, we get

$$
\begin{aligned}
& u_{11}=-2 \frac{\mu^{2}}{r^{3}} \\
& u_{22}=96 \frac{\mu_{\mathrm{q}}^{2} \delta^{2}}{r^{5}} \\
& u_{33}=-2880 \frac{\mu_{\mathrm{q}}^{2} \delta^{4}}{r^{7}} \\
& u_{12}=12 \frac{\mu \mu_{\mathrm{q}} \delta}{r^{4}} \\
& u_{13}=-48 \frac{\mu \mu_{\mathrm{q}} \delta^{2}}{r^{5}} .
\end{aligned}
$$

\section{References}

1. Karlström, G., Linse, P.: J. Stat. Phys. (Submitted). doi:10.1007/s10955-011-0353-1 
2. Verlet, L., Weis, J.J.: Mol. Phys. 28, 665 (1974)

3. Patey, G.N., Valleau, J.P.: J. Chem. Phys. 64, 170 (1976)

4. Levesque, D., Patey, G.N., Weis, J.J.: Mol. Phys. 34, 1077 (1977)

5. Ladd, A.J.C.: Mol. Phys. 36, 463 (1978)

6. Wang, S.S., Gray, C.G., Egelstaff, P.A., Gubbins, K.E.: Chem. Phys. Lett. 21, 123 (1973)

7. McDonald, I.R.: J. Phys. C 7, 1225 (1974)

8. Morriss, G.P.: Mol. Phys. 47, 833-848 (1982)

9. Evans, D.F., Wennerström, H.: The Colloidal Domain: Where Physics, Chemistry, Biology and Technology Meet, 2nd edn. Wiley-VCH, New York (1999)

10. Stenhammar, J., Linse, P., Karlström, G.: Mol. Phys. 109, 11-20 (2011), and references given therein

11. Berendsen, H.J.C., Postma, J.P.M., van Gunsteren, W.F., DiNola, A., Haak, J.R.: J. Chem. Phys. 81, 3684 (1984)

12. MOLSIM, Version 4.9, Linse P. Lund University, Sweden (2010)

13. Karlström, G., Stenhammar, J., Linse, P.: J. Phys. Condens. Matter 20, 494204-1-494204-9 (2008)

14. Allan, M.P., Tildesley, D.J.: Computer Simulations of Liquids. Clarendon Press, Oxford (1986) 